\title{
On Computing the Minimal Number of Defining Relations for Finite Groups
}

\author{
By T. W. Sag and J. W. Wamsley
}

\begin{abstract}
This paper describes a method for computing the Schur multiplicator of a finite supersolvable group $G$, given by some fixed generating system chosen from a cyclic series for $G$, and hence a lower bound for the minimal number of relations needed to define $G$.
\end{abstract}

1. Introduction. This paper describes a method for computing a lower bound for the minimal number of defining relations for finite supersolvable groups. It is implicit in Schur [1] that a lower bound is given by $n+m$, where $m$ is the minimal number of generators of the Schur multiplicator as an abelian group and $n$ is the number of generators of the group. It is known that in general this bound is not exact; however, in the case of finite nilpotent groups, it appears that the bound is reasonable and no example is known that shows it not exact.

The finite groups considered here have a presentation of the form

$$
\begin{aligned}
G=\left\{a_{1}, \cdots, a_{n} \mid\right. & a_{i} a_{j} a_{i}^{-1} a_{i-1}^{-\beta_{i-1, j}} \cdots a_{1}^{-\beta_{1 j}}, \\
& \left.a_{k}^{\rho_{k}} a_{k-1}^{-\gamma_{k-1}} \cdots a_{1}^{-\gamma_{1}}, 1 \leqq j<i \leqq n, 1 \leqq k \leqq n\right\}=F / R,
\end{aligned}
$$

where $F$ is the free group on $a_{1}, \cdots, a_{n}$ and $R$ is the smallest normal subgroup of $F$ containing the given relators. Let $T$ be the set of these relators.

It is generally the case that less than $n(n+1) / 2$ (the total number of elements of $T$ ) relators are required to define $G$ and the method given calculates a lower bound for the number required, by computing the minimal number of generators of $R /[F, R]$. We do this by starting with a set of free generators $\left\{r_{i}\right\}$ for the free abelian group $R /[R, R]$, which may be considered as a $G$-module under conjugation, whence $R /[F, R]$ is the abelian group generated by $\left\{r_{i}\right\}$ with relations

$$
a_{k} r_{i} a_{k}^{-1}=r_{i}, \quad 1 \leqq k \leqq n,
$$

where the left-hand side of (2) is first expanded in terms of free generators of $R /[R, R]$.

However, the number of relations produced in this way is large; but by carrying out the operations in a certain order, we are able to end only with relations between the relators in the set $T$ rather than relations between the whole set $\left\{r_{i}\right\}$.

2. Mathematical Details. Let $G$ be a group with presentation given in (1), then we may choose a set of coset representatives of $R$ in $F$ :

Rec eived January 20, 1972, revised July 14, 1972.

AMS (MOS) subject classifications (1970). Primary 20F05, 20D15. minimal.

Key words and phrases. Finite supersolvable groups, relations, generators, Schur multiplicator, 


$$
\left\{a_{1}^{\alpha_{1}} \cdots a_{n}^{\alpha_{n}} \mid 0 \leqq \alpha_{i}<\rho_{i}\right\} \text { (see for example Hall [2, Chapter 7]) }
$$

whence $R$ is freely generated by the set

$$
\left\{\left(a_{1}^{\alpha_{1}} \cdots a_{n}^{\alpha_{n}} a_{j}\right)^{*} \neq I\right\}, \quad \text { where } I \text { is the identity, }
$$

$\left(g a_{j}\right)^{*}$ denotes $g a_{j}\left[g a_{j}\right]^{-1}$ and $\left[g a_{j}\right]$ denotes the coset representative of $g a_{i}$. This yields the following set of free generators for $R /[R, R]$ :

(4) $S=\left\{\begin{array}{lc}\left(a_{1}^{\alpha_{1}} \cdots a_{i-1}^{\alpha_{i-1}} a_{i} a_{j}\right)^{*}, j=1, \cdots, i-1 & 0 \leqq \alpha_{k}<\rho_{k}, \\ \left(a_{1}^{\alpha_{i}} \cdots a_{i-1}^{\alpha_{i-1}} a_{i}^{\rho_{i}}\right)^{*} & k=1, \cdots, i-1, \\ \left(a_{1}^{\alpha_{1}} \cdots a_{i}^{\alpha_{i}} a_{j}\right)^{*}, j=1, \cdots, i-1 & 0 \leqq \alpha_{k}<\rho_{k}, \\ & k=1, \cdots, i-1, \\ & 2 \leqq \alpha_{i}<\rho_{i} .\end{array}\right\}$

If we take an arbitrary element $s$ of $S$ and conjugate it with $a_{k}$, then, using an algorithm given by Hall [2], we can express $a_{k} s a_{k}^{-1}$ in terms of elements of the set $S$. In particular, if we take $s$ to be of the form

$$
s=\left(a_{k}^{\alpha_{k-1}} a_{k+1}^{\alpha_{k+1}} \cdots a_{i}^{\alpha_{i}} a_{j}\right)^{*},
$$

we have

$$
\begin{gathered}
a_{k}\left(a_{k}^{\alpha_{k-1}} a_{k+1}^{\alpha_{k+1}} \cdots a_{i}^{\alpha_{i}} a_{j}\right)^{*} a_{k}^{-1} \\
=a_{k} a_{k}^{\alpha_{k}-1} a_{k+1}^{\alpha_{k+1}} \cdots a_{i}^{\alpha_{i}} a_{i}\left[a_{k}^{\alpha_{k}-1} a_{k+1}^{\alpha_{k+1}} \cdots a_{i}^{\alpha_{i}} a_{j}\right]^{-1} a_{k}^{-1} \\
=a_{k}^{\alpha_{k}} a_{k+1}^{\alpha_{k+1}} \cdots a_{i}^{\alpha_{i}} a_{j}\left[a_{k}^{\alpha_{k}} \cdots a_{i}^{\alpha_{i}} a_{j}\right]^{-1}\left[a_{k}^{\alpha_{k}} \cdots a_{i}^{\alpha_{i}} a_{j}\right] . \\
{\left[\begin{array}{rl}
a_{k}^{\alpha_{k}-1} a_{k+1}^{\alpha_{k+1}} \cdots & \left.a_{i}^{\alpha_{i}} a_{j}\right]^{-1} a_{k}^{-1} \\
= & \left(a_{k}^{\alpha_{k}} \cdots a_{i}^{\alpha_{i}} a_{j}\right)^{*}\left[a_{k}^{\alpha_{k}} \cdots a_{i}^{\alpha_{i}} a_{j}\right]\left[a_{k}^{\alpha_{k}-1} a_{k+1}^{\alpha_{k+1}} \cdots a_{i}^{\alpha_{i}} a_{j}\right]^{-1} a_{k}^{-1} .
\end{array}\right.}
\end{gathered}
$$

Now

$$
\begin{aligned}
{\left[a_{k}^{\alpha_{k}} \cdots a_{i}^{\alpha_{i}} a_{i}\right] } & {\left[a_{k}^{\alpha_{k-1}} a_{k+1}^{\alpha_{k+1}} \cdots a_{i}^{\alpha_{i}} a_{i}\right]^{-1} a_{k}^{-1} } \\
& =\left(a_{k}\left[a_{k}^{\alpha_{k-1}} a_{k+1}^{\alpha_{k+1}} \cdots a_{i}^{\alpha_{i}} a_{j}\right]\left[a_{k}^{\alpha_{k}} \cdots a_{i}^{\alpha_{i}} a_{j}\right]^{-1}\right)^{-1} \\
& =\left\{\prod_{l=1, k-1, \beta l \neq 0 ; m=0, \beta_{l}-1}\left(\left[a_{k} a_{1}^{\beta_{1}} \cdots a_{l-1}^{\beta_{l}-1} a_{l}^{m}\right] a_{l}\right)^{*} \cdot e\right\}^{-1},
\end{aligned}
$$

where $\beta_{1}, \cdots, \beta_{k}$ are such that

$$
a_{1}^{\beta_{1}} \cdots a_{k}^{\beta_{k}}=\left[a_{k}^{\alpha_{k-1}} a_{k+1}^{\alpha_{k+1}} \cdots a_{i}^{\alpha_{i}} a_{i}\right]
$$

and

$$
\begin{aligned}
& e=\left(\left[a_{k} a_{1}^{\beta_{1}} \cdots a_{k}^{\beta_{k-1}}\right] a_{k}\right)^{*} \text { if } \beta_{k}=\rho_{k}-1 \text { and } \\
& e=I \text { otherwise, }
\end{aligned}
$$

Hence, we have

$$
\left(a_{k}^{\alpha_{k}} \cdots a_{i}^{\alpha_{i}} a_{j}\right)^{*}=a_{k}\left(a_{k}^{\alpha_{k-1}} a_{k+1}^{\alpha_{k+1}} \cdots a_{i}^{\alpha_{i}} a_{j}\right)^{*} a_{k}^{-1}
$$

$$
\cdot \prod_{l=1, k-1, \beta l \neq 0 ; m=0, \beta_{l}-1}\left(\left[a_{k} a_{1}^{\beta_{1}} \cdots a_{l-1}^{\beta_{l-1}} a_{l}^{m}\right] a_{l}\right)^{*} \cdot e .
$$


A similar result can be obtained for elements of the second type in (4) by setting $\alpha_{i}=\rho_{i}-1$ and $j=i$. If the elements in $S$ are numbered in the order given in (4), then the elements of $S$ occurring under the product sign and the element $e$ if $e \neq I$ are found to have lower numbers than the element on the left-hand side of (5). If one applies (5) to the elements of $S$ in the order they are listed in (4) and substitutes earlier results in the right-hand side, one can express the elements of $S$ in terms of the elements

$$
\begin{array}{ll}
a_{1}^{\alpha_{1}} \cdots a_{i-1}^{\alpha_{i-1}}\left(a_{i}^{\rho_{i}}\right)^{*} a_{i-1}^{-\alpha_{i-1}} \cdots a_{1}^{-\alpha_{1}}, & 0<i \leqq n, \\
a_{1}^{\alpha_{1}} \cdots a_{i}^{\alpha_{i-1}}\left(a_{i} a_{i}\right)^{*} a_{i}^{1-\alpha_{i}} \cdots a_{1}^{-\alpha_{1}}, & 0<j<i \leqq n .
\end{array}
$$

Hence, the set (6) generates $R /[R, R]$, and since the number of elements in (6) is the same as the number of elements in (4), the set (6) is a set of free generators for the free abelian group $R /[R, R]$.

On applying the relations (2) to the set (6), we find first that elements of this set reduce respectively to

$$
\begin{array}{ll}
\left(a_{i}^{\rho_{i}}\right)^{*}, & 0<i \leqq n, \\
\left(a_{i} a_{j}\right)^{*}, & 0<j<i \leqq n,
\end{array}
$$

which are precisely the relators in the set $T$ given in (1). Secondly, if we denote $\mathrm{gsg}^{-1}$ by $g \circ s$, then, since $R /[R, R]$ is a $G$-module, we have

$$
a_{k} \circ\left\{a_{1}^{\alpha_{1}} \cdots a_{i-1}^{\alpha_{i-1}} \circ\left(a_{i}^{\rho_{i}}\right)^{*}\right\}=\left[a_{k} a_{1}^{\alpha_{1}} \cdots a_{i-1}^{\alpha_{i-1}}\right] \circ\left(a_{i}^{\rho_{i}}\right)^{*}
$$

and, in the case $k<i$, the right-hand side reduces to the form

$$
\left(a_{1}^{\beta_{1}} \cdots a_{l}^{\beta_{l}}\right) \circ\left(a_{i}^{\rho_{i}}\right)^{*} \text { for some } l<i
$$

which is just an element of the set (6). A similar result is obtained if $a_{i}^{\rho_{i}}$ is replaced by $a_{i} a_{i}$ for $j<i$. Thus, nontrivial relations may only be obtained in the case $k \geqq i$. Hence, $R /[F, R]$ is the abelian group with generators given by (7) and the following relations:

$$
\begin{aligned}
a_{i} \circ\left(a_{i} a_{k}\right)^{*} & =\left(a_{i} a_{k}\right)^{*}, & & 1 \leqq k<j<i \leqq n, \\
a_{i} \circ\left(a_{i}^{\rho_{i}-1} a_{k}\right)^{*} & =\left(a_{i}^{\rho_{i}-1} a_{k}\right)^{*}, & & 1 \leqq k<i \leqq n, \\
a_{i} \circ\left(a_{i}^{\rho_{i}}\right)^{*} & =\left(a_{i}^{\rho_{i}}\right)^{*}, & & 1 \leqq j<i \leqq n, \\
a_{i} \circ\left(a_{i}^{\rho_{i}}\right)^{*} & =\left(a_{i}^{\rho_{i}}\right)^{*}, & & 1 \leqq i \leqq n .
\end{aligned}
$$

The terms in relations (8) to (11), when expressed in terms of free generators of the form (4), yield the following relations:

$$
\begin{aligned}
\left(a_{i} a_{k}\right)^{*} \cdot \prod_{l=1, i, \beta_{l} \neq 0 ; m=0, \beta_{l}-1}\left(\left[a_{i} a_{1}^{\beta_{1}} \cdots a_{l-1}^{\beta_{l}-1} a_{l}^{m}\right] a_{l}\right)^{*} \\
\cdot\left(a_{i} a_{j}\right)^{*-1} \cdot\left(\left[a_{i} a_{j}\right] a_{k}\right)^{*^{-1}}=I \text { for } 1 \leqq k<j<i \leqq n,
\end{aligned}
$$

where $\left[a_{i} a_{k}\right]=a_{1}^{\beta_{1}} \cdots a_{i}^{\beta_{i}}$,

$$
\begin{aligned}
\left(a_{i}^{\rho_{i}-1} a_{k}\right)^{*} . \prod_{l=1, i-1, \beta l \neq 0 ; m=0, \beta_{l}-1}\left(\left[a_{i} a_{1}^{\beta_{1}} \cdots a_{l-1}^{\beta_{l}-1} a_{l}^{m}\right] a_{l}\right)^{*} \\
\cdot\left(\left[a_{i} a_{1}^{\beta_{1}} \cdots a_{i-1}^{\beta_{i}-1} a_{i}^{\beta_{i}-1}\right] a_{i}\right)^{*} \cdot\left(a_{i}^{\rho_{i}}\right)^{*^{-1}} \cdot\left(\left[a_{i}^{\rho_{i}}\right] a_{k}\right)^{*-1}=I
\end{aligned}
$$


for $1 \leqq k<i \leqq n$ where $\left[a_{i}^{\rho_{i}-1} a_{k}\right]=a_{1}^{\beta_{1}} \cdots a_{i}^{\beta_{i}}$. Note that the term $\left(\left[a_{i}^{\rho_{i}}\right] a_{k}\right)^{*}$ may equal $I$, in which case it is omitted.

$$
\begin{aligned}
\left(a_{i}^{\rho_{j}}\right)^{*} \cdot \prod_{l=1, i-1, \beta_{l \neq 0 ; m=0, \beta_{l}-1}}\left(\left[a_{i} a_{1}^{\beta_{1}} \cdots a_{l-1}^{\beta_{l}-1} a_{l}^{m}\right] a_{l}\right)^{*} & \\
& \cdot \prod_{m=0, \rho_{j}-1}\left(\left[a_{i} a_{i}^{m}\right] a_{j}\right)^{*-1}=I \quad \text { for } 1 \leqq j<i \leqq n
\end{aligned}
$$

where $\left[a_{i}^{\rho_{i}}\right]=a_{1}^{\beta_{1}} \cdots a_{i-1}^{\beta_{i-1}}$,

$$
\prod_{l=1, i-1, \beta l \neq 0 ; m=0, \beta_{l}-1}\left(\left[a_{i} a_{1}^{\beta_{1}} \cdots a_{l-1}^{\beta_{l-1}} a_{l}^{m}\right] a_{l}\right)^{*}=I \quad \text { for } 1 \leqq i \leqq n
$$

where

$$
\left[a_{i}^{\rho_{i}}\right]=a_{1}^{\beta_{1}} \cdots a_{i-1}^{\beta_{i}-1} .
$$

Using the results of the repeated application of (5), the left-hand sides of relations (12) to (15) can be converted to products of powers of elements of the form listed in (6) and, by virtue of the result of applying (2) to these elements, one finally obtains relations involving products of powers of the relators in the set $T$. In this way, we obtain a presentation for $R /[F, R]$ given on the $N=n(n+1) / 2$ relators in $T$ with $M=\left(n^{3}+3 n^{2}+2 n\right) / 6$ relations of the form

$$
\prod_{k=1}^{N} t_{k}^{\alpha_{i k}}=I, \quad j=1, \cdots, M,
$$

where the $t_{k}$ are the elements of $T$.

By performing a unimodular diagonalization of the integer matrix with elements $\alpha_{i k}$, one can obtain an equivalent set of relations of the form

$$
s_{i}^{\delta_{i}}=I, \quad i=1, \cdots, N,
$$

where the $\delta_{i}$ are the diagonal elements of the resulting diagonal matrix and the $s_{i}$ are products of powers of the $t_{k}$, the exponents of the $t_{k}$ depending on the operations performed in the diagonalization. The group $R /[F, R]$ is then precisely the direct product $C_{\delta_{1}} \times C_{\delta_{2}} \times \cdots \times C_{\delta_{N}}$ where $C_{\delta}$ is the cyclic group of order $\delta$.

3. Outline of Computer Program. The computer program calculates the numbers $\delta_{i}$ in Eq. (17), the computation being carried out in three stages.

In the first stage, the exponents of the relators in $T$, which occur when the elements of $S$ are expressed as products of powers of the relators, are computed. For each element $\left(g a_{i}\right)^{*}$ in $S$, in the order specified by (4), the following computations are carried out.

First, numbers $\beta_{1}, \cdots, \beta_{n}$ such that $\left[g a_{j}\right]=a_{1}^{\beta_{1}} \cdots a_{n}^{\beta_{n}}$ are found. In cases where $\left(g a_{i}\right)^{*}$ is an element of $T$, these numbers are obtainable directly as the exponents $\beta_{1 i}, \cdots, \beta_{i-1, i}$ or $\gamma_{1}, \cdots, \gamma_{k-1}$ in the appropriate relator in (1). In other cases, the numbers can be obtained by performing multiplication of $g$ with $a_{j}$ in $G$ by using the fact that the relators are equal to the identity to expand $a_{i} a_{i}, j<i$, and $a_{i}^{\gamma}, \gamma \geqq \rho_{i}$ when they occur in the product. Secondly, the required exponents $\theta_{1}, \cdots, \theta_{N}$ of relators $t_{1}, \cdots, t_{N}$ in the product of powers of relators for the element are found as 
follows. If the element is a relator (say $t_{k}$ ), then $\theta_{k}$ is set equal to 1 , and all other exponents are set equal to zero. Otherwise, the elements of $S$ occurring on the righthand side of the appropriate relation (5) are found successively and the exponents of relators in $T$ associated with these elements are successively summed to give the exponents of relators for the element of $S$ on the left-hand side of (5).

In the second stage, the numbers $\alpha_{j k}$ in (16) are found by finding the elements of $S$ occurring on the left-hand sides of the relations (12) to (15) for the appropriate values of $i$, $j$, and $k$, and, in each case, summing the exponents of the relators associated with these elements.

In the third stage, the matrix consisting of the nonzero rows of the matrix $\left[\alpha_{i k}\right]$ is diagonalized by performing a unimodular diagonalization (for example see Bradley [3]) to obtain the required $\delta_{i}$ as diagonal elements.

Further details of certain parts of the program are given in the following sections.

4. Numbering System for Elements of $S$. In the program, a number $\eta(s)$ is assigned to each element $s$ of $S$. This number indicates its position in the list (4) and is also used as an index for storing numbers associated with the element such as the numbers $\beta_{1}, \cdots, \beta_{n}$ and the exponents $\theta_{1}, \cdots, \theta_{N}$. This number can also be used to determine $j$ and the coset representative of $g$ where $s=\left(g a_{i}\right)^{*}$. The numbers for the three types of elements in the list (4) are found to be

$$
\begin{aligned}
& \eta\left(\left(a_{1}^{\alpha_{1}} \cdots a_{i-1}^{\alpha_{i-1}} a_{i} a_{i}\right)^{*}\right) \\
& =N_{i}+i\left(\alpha_{1}+\rho_{1} \alpha_{2}+\cdots+\rho_{1} \rho_{2} \cdots \rho_{i-2} \alpha_{i-1}\right)+j \text { for } j<i \text {, } \\
& \eta\left(\left(a_{1}^{\alpha_{1}} \cdots a_{i-1}^{\alpha_{i-1}} a_{i}^{\rho_{i}}\right)^{*}\right)=N_{i}+i\left(\alpha_{1}+\rho_{1} \alpha_{2}+\cdots+\rho_{1} \rho_{2} \cdots \rho_{i-2} \alpha_{i-1}\right)+i \text {, } \\
& \eta\left(\left(a_{1}^{\alpha_{1}} \cdots a_{i-1}^{\alpha_{i-1}} a_{i}^{\alpha_{i}} a_{i}\right)^{*}\right) \\
& =N_{i}+(i-1)\left(\alpha_{1}+\rho_{1} \alpha_{2}+\cdots+\rho_{1} \rho_{2} \cdots \rho_{i-1}\left(\alpha_{i}-1\right)\right) \\
& +\rho_{1} \rho_{2} \cdots \rho_{i-1}+j \text { for } j<i \text { and } 2 \leqq \alpha_{i}<\rho_{i} \text {, }
\end{aligned}
$$

where $N_{i}$, the number of elements of $S$ involving generators selected from $a_{1}, \cdots, a_{i-1}$, is given by

$$
\begin{aligned}
& N_{1}=0, \quad N_{2}=1 \\
& N_{i}=N_{i-1}+(i-2) \rho_{1} \rho_{2} \cdots \rho_{i-2}\left(\rho_{i-1}-1\right)+\rho_{1} \rho_{2} \cdots \rho_{i-2}, \quad i>2 .
\end{aligned}
$$

5. Performing Group Multiplication. In order to compute the numbers $\beta_{1}, \cdots, \beta_{n}$ associated with an element $\left(g a_{i}\right)^{*}$ of $S$, it is sometimes neccessary to multiply $g$ with $a_{i}$ and hence obtain the coset representative of $g a_{i}$. Given the coset representative of $g$, say $g=a_{i 1}^{\alpha_{i 1}} a_{i 2}^{\alpha_{i 2}} \cdots a_{i k}^{\alpha_{i k}}$, and given the exponents $\beta_{1 i}, \cdots, \beta_{i-1, i}$ and $\gamma_{1}, \cdots, \gamma_{k-1}$ associated with the relators in (1) (these are fed in as data to the program), this is done in the following way.

First, two arrays with elements, say $C_{r_{1}}$ and $C_{r 2}$, are set up to contain the nonzero exponents of the generators $a_{1}, \cdots, a_{n}$ and the indices of the corresponding generators, respectively, in the order in which they occur in the product $g a_{j}$. Thus, initially, we have

$$
\left(C_{11}, \cdots, C_{k+1,1}\right)=\left(\alpha_{i 1}, \cdots, \alpha_{i k}, 1\right)
$$


and

$$
\left(C_{12}, \cdots, C_{k+1,2}\right)=(i 1, \cdots, i k, j) .
$$

The total number of elements $m(k+1$ in this case) in each array is noted, and then, for $r=2, \cdots, m$, the indices $p=C_{r-1,2}$ and $q=C_{r 2}$ are compared. If $p<q$, then the next pair of indices are compared.

If $p=q$, then $d=C_{r-1,1}+C_{r 1}$ is computed and compared with $\rho_{p}$. (Note $\rho_{1}, \cdots, \rho_{n}$ are fed in as data to the program.) If $d<\rho_{p}$, then $C_{r-1,1}$ is set equal to $d$, and the elements $C_{r 1}$ and $C_{r 2}$ are removed from the arrays by shifting the elements $C_{i 1}$ and $C_{i 2}$ one place to the left for $i=r+1, \cdots, m$, and $m$ is decreased by 1 . If $d=\rho_{p}$, then the elements $C_{r-1,1}, C_{r 1}, C_{r-1,2}$ and $C_{r 2}$ are replaced by the nonzero exponents selected from the exponents $\gamma_{1}, \cdots, \gamma_{p-1}$ associated with the relator $\left(a_{p}^{\rho_{p}}\right)^{*}$, and the corresponding indices selected from $1, \cdots, p-1$, respectively, and the new number $m$ of elements in each of the two arrays is computed. If $d>\rho_{p}$, then $C_{r-1,1}, C_{r 1}, C_{r-1,2}$ and $C_{r 2}$ are replaced by these exponents and indices followed by the exponent $d-\rho_{p}$ and the index $p$.

If $p>q$, then the nonzero exponents selected from the $\beta_{1 q}, \cdots, \beta_{p-1, q}$ and $\beta_{p q}=1$ preceded by the exponent $C_{r-1,1}-1$ (if $C_{r-1,1}>1$ ) and followed by $C_{r 1}-1$ (if $C_{r 1}>1$ ), and the corresponding indices selected from $1, \cdots, p$ preceded by $p$ and followed by $q$ (if appropriate) are respectively inserted in the two arrays in place of $C_{r-1,1}, C_{r 1}$, and $C_{r-1,2}$ and $C_{r 2}$, respectively, and the total number of elements $m$ in each array is updated.

In each of the cases, $p=q$ and $p>q$, after the arrays have been changed, the indices $C_{r-1,2}$ and $C_{r 2}$ are compared from the beginning again. The process terminates when the indices $C_{r 2}, r=1, \cdots, m$, are in ascending order. The array $C_{r 1}$ then contains the nonzero $\beta_{1}, \cdots, \beta_{n}$ and the array $C_{r 2}$ contains the corresponding indices of the generators. This enables $\beta_{1}, \cdots, \beta_{n}$ to be found.

6. Determining the Elements of $S$ Occurring in Products. In order to compute the exponents $\theta_{1}, \cdots, \theta_{N}$ of relators in the expression of elements of $S$ in terms of relators, and in order to compute the exponents $\alpha_{j k}$ in (16), it is neccessary to find the numbers $\eta(s)$ of the elements $s$ of $S$ occurring in the products on the righthand side of (5) and the left-hand sides of (12) to (15). This is facilitated by computing for each element $s$ of $S$ a number $\bar{\eta}(s)$ which is computed from the numbers $\beta_{1}, \cdots, \beta_{n}$ corresponding to $s$. The numbers $\bar{\eta}(s)$ are such that if $s=\left(a_{1}^{\beta_{1}} \cdots a_{i}^{\beta_{i}}\right)^{*}=(g)^{*}$ and $\bar{s}=\left(g a_{i}\right)^{*}$ then $\eta(\bar{s})=\bar{\eta}(s)+j$ for $j<i$. Bearing in mind the numbering system given by (18), the required $\eta(s)$ are found to be

$$
\bar{\eta}(s)=N_{i}+i\left(\beta_{1}+\rho_{1} \beta_{2}+\cdots+\rho_{1} \rho_{2} \cdots \rho_{i-2} \beta_{i-1}\right) \quad \text { if } \beta_{i}=1
$$

and

$$
\begin{aligned}
\bar{\eta}(s)= & N_{i}+(i-1)\left(\beta_{1}+\rho_{1} \beta_{2}+\cdots+\rho_{1} \rho_{2} \cdots \rho_{i-1}\left(\beta_{i}-1\right)\right) \\
& +\rho_{1} \rho_{2} \cdots \rho_{i-1} \quad \text { if } \beta_{i}>1
\end{aligned}
$$

and

$$
\bar{\eta}(s)=0 \quad \text { if } i=1 \text { and } \beta_{1}=\rho_{1}-1
$$


Note, in the case $i=1, \beta_{1}<\rho_{1}-1, \bar{\eta}(s)$ is not required but may be set equal to, say, -1 for convenience.

The numbers $\eta(s)$ of the elements $s$ occurring on the right-hand side of (5) can now be computed as follows.

First, since elements of $S$ are expressible as products of powers of the elements listed in (6), which in turn reduce to the relators in (7), the first element in the product on the right-hand side reduces to

$$
s_{1}=\left(a_{k}^{\alpha_{k}-1} a_{k+1}^{\alpha_{k+1}} \cdots a_{i}^{\alpha_{i}} a_{j}\right)^{*}
$$

and its number $\eta\left(s_{1}\right)$ is given in terms of $\eta(s)$, where

$$
s=\left(a_{k}^{\alpha_{k} k} a_{k+1}^{\alpha_{k+1}} \cdots a_{i}^{\alpha_{i}} a_{i}\right)^{*}
$$

as follows:

If $k<i$, then

$$
\eta\left(s_{1}\right)=\eta(s)-i \times \rho_{1} \rho_{2} \cdots \rho_{k-1} \quad \text { if } \alpha_{i}=1,
$$

and

$$
\eta\left(s_{1}\right)=\eta(s)-(i-1) \rho_{1} \rho_{2} \cdots \rho_{k-1} \quad \text { if } \alpha_{i}>1 .
$$

If $k=i$, then $\eta\left(s_{1}\right)$ is given by the first of the above forms if $\alpha_{i}=2$, and the second form if $\alpha_{i}>2$. (Note in the case $\alpha_{i}=1, s=\left(a_{i} a_{i}\right)^{*}$.)

The numbers $\beta_{1}, \cdots, \beta_{k}$ occurring in the remainder of the product can now be found as these are the $\beta$ 's associated with $s_{1}$. This enables one to find the next element in the product which is $s_{2}=\left(a_{k} a_{q}\right)^{*}$ where $q$ is such that $\beta_{q}$ is the first nonzero $\beta$ among $\beta_{1}, \cdots, \beta_{k}$. Its number $\eta\left(s_{2}\right)$ is obviously $N_{k}+q$. The numbers $\eta\left(s_{r}\right)$ of the remaining elements $s_{r}$ in the product, including the element $e$ if $e \neq I$ and $\beta_{1}, \cdots, \beta_{k-1}$ are not all zero, can now be found by the formula

$$
\eta\left(s_{r}\right)=\eta\left(s_{r-1}\right)+l,
$$

where $l$ is such that

$$
s_{r}=\left(g_{r-1} a_{l}\right)^{*} \text { where } s_{r-1}=\left(g_{r-1}\right)^{*} .
$$

If $\beta_{1}, \cdots, \beta_{k-1}$ are all zero and $\beta_{k}=\rho_{k}-1$, then $e=\left(a_{k}^{\rho k}\right)^{*}$ and hence

$$
\eta(e)=N_{k}+k \text {. }
$$

The numbers of the elements occurring in the products on the left-hand sides of relations (12) to (15) are computed by using (18) directly for elements which involve only one or two generators, and then using a process similar to that described above to compute the numbers for the more complicated elements. 
7. Groups with a Common Stem. If the relators for two successive groups $G_{1}$ and $G_{2}$, involving the generators $a_{1}, \cdots, a_{n 1}$, are the same, then the exponents $\theta_{1}, \cdots, \theta_{N}$ for the elements of $S$ involving only some or all of these generators, and the exponents $\alpha_{j k}$ in (16) obtained from relations (12) to (15) in the cases $i \leqq n 1$, will be the same for both $G_{1}$ and $G_{2}$. Advantage of this fact is taken in the program by arranging for it to carry out the computations for stages 1 and 2 only for $i=n 1+$ $1, \cdots, n$ in the case of $G_{2}$. This is facilitated by feeding in $n 1$ and $n$ as input data and allowing the program to use the information previously computed for $i=1, \cdots, n 1$.

8. Conclusion. The program described above was written in FORTRAN and run on the CDC 6400 computer at the University of Adelaide. It has been used successfully to determine lower bounds for the minimal number of defining relations for all groups of order $2^{n}, n \leqq 6$, which are listed in Hall and Senior [4]. This enabled minimal presentations to be found for these groups, and these are given in Sag and Wamsley [5]. A similar computation has also been carried out for groups of order $3^{n}, n \leqq 6$, and the method has also been used by Wamsley [6] to calculate by hand a minimal presentation for a general wreath product of two $p$-groups given minimal presentations for each of the two groups. Computing time per group averaged 3 seconds for the groups of order $2^{n}, n \leqq 6$, and 12 seconds for the groups of order $3^{n}, n \leqq 6$.

School of Mathematical Sciences

Flinders University of South Australia

Bedford Park, South Australia 5042

1. J. Schur, "Untersuchungen über die Darstellung der endlichen Gruppen durch gebrochene lineare Substitutionen," J. Reine Angew. Math., v. 132, 1907, pp. 85-137.

2. M. Hall, JR., The Theory of Groups, Macmillan, New York, 1959. MR 21 \#1996.

3. G. H. BRadLeY, "Algorithms for Hermite and Smith normal form matrices," Math. Comp., v. 25, 1971, pp. 897-907.

4. M. HALl, JR. \& J. K. Senior, The Groups of Order $2^{n}$, $(n \leqq 6)$, Macmillan, New York, 1964. MR 29 \#5889.

5. T. W. SAG \& J. W. WAMSLEY, "Minimal presentations for groups of order $2^{n}, n \leqq 6$," J. Austral. Math. Soc. (To appear.)

6. J. W. WAMSLEY, "The deficiency of wreath products of groups," J. Algebra. (To appear.) 